\title{
Genome-centered integrated instrumental information system modeling and interpretation of human and virus omics
}

\author{
Anatoliy Shlikht \\ dept. life safety \\ Far Eastern Federal University \\ Vladivostok, Russia \\ schliht@mail.ru
}

\author{
Natalia Kramorenko \\ dept. business informatics and economic-mathematical methods \\ Far Eastern Federal University \\ Vladivostok, Russia \\ kramnat@mail.ru
}

\begin{abstract}
Creating an integrated genome-centered intellectual system based on highly structured databases and knowledge bases for modeling and interpreting human and virus omics. The system allows you to automatically find functionally significant structures (templates, motifs, epitopes) for genomic and proteomic data in the formation of nucleoprotein complexes and antigen-antibody complexes, determine protein functions, and model spatio-temporal biochemical and physiological processes.
\end{abstract}

Keywords - genomics, proteomics, databases, knowledge bases, modeling, interpretation

\section{Introduction}

Genome-centered analysis and interpretation faces the challenges of storing, searching, and modeling big omics data (genomics, transcriptomics, proteomics, metabolomics, and others). This fully applies to the tasks of viral infections. When solving problems of virology (molecular diagnostics, vaccination, search for therapeutic drugs), it is necessary to move from big omics data to functionally significant molecular structures: mutations, motives, conservative domains, enzymes, metabolic reactions and pathways, physiological systems, diseases based on bioinformatics technologies. This approach allows us to build a complete picture of the biochemical and physiological processes in a living organism, in particular, when the human organism interacts with viruses.

\section{Methods and Algorithms}

The goal of this work is to create an integrated genomecentered intellectual system based on highly structured databases and knowledge bases for processing omics data in the tasks of integrating genomic virology with the human genome.

Currently existing big weakly structured omics data from international portals (NCBI, KEGG, Ensembl, HGNC, etc.) with interactive data access technologies do not allow to effectively solve the problems of integration of genomic virology and the human genome. An effective solution to these problems can be obtained and associated with the creation of highly structured indexed omics databases and knowledge bases, followed by automatic analysis and interpretation of primary data based on expert systems technology. It is necessary to restructure primary data as much as possible, automate interactive scenarios, shifting them to intelligent expert systems.

\section{Results}

Highly structured indexed models of omics data of numerous viruses and the reference human genome have been developed [1,2], on the basis of which databases and knowledge bases have been created, which form the basis of expert systems for automatic analysis, interpretation and diagnosis of viral diseases.

The expert system (ES) has been created that automatically searches for functionally significant structures (patterns, motifs, epitopes) for both genomic data and amino acid sequences of protein in the formation of nucleo-protein complexes and antigen-antibody complexes.

Special filters have been developed that work both on the basis of Prosite patterns (https://prosite.expasy.org/) and arbitrary templates, allowing you to find functionally significant domains and define protein functions. For automatic operation of these filters, a special compiler has been developed that allows modeling various search structures and templates.

The ES automatically searches for mutations for the studied genes and proteins. ES allow efficient automatic analysis and interpretation of genomic and proteomic data, and subsequent diagnosis of diseases.

A distinctive feature of the developed ES is their Autonomous nature, which allows you to work autonomously with both the reference human genome and virus genomes without constantly accessing data from world portals. At the same time, the mode of periodic updating of omics data from world portals for data updating is provided.

The created ES allow us to trace deep connections, starting from a mutation in the gene along the chain: (DNA, RNA) gene - information RNA - encoded protein - enzyme metabolic reaction - metabolic pathway - physiological system - disease. This view allows the medic, biologist, geneticist, and researcher to trace the entire depth of biological and physiological processes. Created databases and knowledge bases allow you to determine the genotype of humans and a virus.

For the convenience of working with the developed systems (databases and knowledge bases), specialists who do not know information technologies have developed a special visual dialog interface. The interface is based on simple graphical constructs of visual programming.

The system can also be used effectively to analyze the results of genomic sequencing with subsequent interpretation of mutations. Based on the developed filters, the most significant mutations and their manifestation in proteins and metabolic processes are identified.

\section{Conclusion}

The developed information tool systems (databases and knowledge bases, ES) make it possible to effectively analyze the primary sequences of DNA, RNA, proteins for humans 
and viruses. The created systems proved their effectiveness in diagnostics, as well as in other tasks of molecular biology: search for motives, sites, epitopes; modeling of transcripts; modeling of proteins; modeling of virus strategies; obtaining large omics quantitative data with subsequent transformation into small qualitative semantic data.

\section{REFERENCES}

[1] A.G.Shlikht, N.V.Kramorenko, "Deep bioinformatics expert system of analysis, modeling and interpretation of omics BigData of the human genome", The 3rd International Symposium "Mathematical Modeling and High-Performance Computing in Bioinformatics, Biomedicine and Biotechnology (MM-HPC-BBB-2018)”. Novosibirsk, 2018. P. 66.

[2] A.G.Shlikht, N.V.Kramorenko, "Artificial intelligence in the problems of analysis and interpretation of omics human data", The 11th International Conference "Bioinformatics of Genome Regulation and StructurelSystems Biology (BGRS\SB-2018)”. Novosibirsk, 2018. P. 78 . 